Research Article

\title{
Effects of Construction Sequences and Volume Loss on Perpendicularly Crossing Tunnels
}

\author{
Yanwei Zang $\mathbb{D}{ }^{1}$ Penglu Gan $\mathbb{D}^{1},{ }^{1,2}$ Jia-jia Yan $\mathbb{D}^{1,2}$ Shiming Liu $\mathbb{D}^{1},{ }^{1}$ and Zihai Yan $\mathbb{D}^{1}$ \\ ${ }^{1}$ PowerChina Huadong Engineering Corporation Limited, Zhejiang Engineering Research Center on Smart Rail Transportation, \\ Hangzhou, China \\ ${ }^{2}$ College of Civil Engineering and Architecture, Zhejiang University, Hangzhou, China
}

Correspondence should be addressed to Penglu Gan; dennis19900828@163.com

Received 22 May 2019; Revised 26 September 2019; Accepted 23 October 2019; Published 17 December 2019

Academic Editor: Flavio Stochino

Copyright (C) 2019 Yanwei Zang et al. This is an open access article distributed under the Creative Commons Attribution License, which permits unrestricted use, distribution, and reproduction in any medium, provided the original work is properly cited.

\begin{abstract}
The number of constructed tunnels has been gradually increasing for the past decades due to rapid development in urban areas. However, the soil-structure interaction problems arising from perpendicularly crossing tunnels attract relatively little research attention in the past. In this study, six three-dimensional finite element analyses were conducted to simulate tunnel excavation nearby a perpendicularly crossing existing tunnel, in an attempt to investigate the effects of construction sequences on crosscutting tunnels. The hypoplastic constitutive model for sand is adopted in the numerical analysis to consider the soil small-strain stiffness. Computed results are presented and discussed in terms of ground surface settlement, displacement and deformation of the existing tunnel, and bending moment induced on the existing tunnel. The stress-transfer mechanism in soil nearby the existing tunnel due to tunnelling is also studied.
\end{abstract}

\section{Introduction}

In recent decades, a variety of approaches have been adopted to study the soil-tunnel interaction, including field observations, physical model tests, empirical/analytical methods and numerical simulations [1-4]. However, most research focused on parallel tunnels and piggyback (vertical twin) tunnels instead of perpendicular tunnels. Also, most research only focused on the effect of volume loss during tunnelling but do not consider the effect of weight loss [5-7]. Mair et al. [2] confirmed that the effect of removal of the weight of the soil in the tunnel is not negligible. Therefore, it is necessary to investigate the significance of simulating weight loss during tunnelling and the effect on the nearby existing tunnel.

Limited research has been reported on perpendicularly crossing tunnels. Kim et al. [8] carried out physical model tests using miniature models under $1 \mathrm{~g}$ conditions to investigate the effects of new tunnel construction on existing tunnels in clay. Parallel (side-by-side) and perpendicular (cross-cutting) tunnel models were tested. Three perpendicular tunnels were installed with one new tunnel beneath an existing tunnel followed by another new tunnel above the existing tunnel. The authors concluded that, in the case of perpendicularly crossing tunnels, significant lining deformations and bending moments were observed during the initial stage of tunnel advancement. However, since the tests were carried out at $1 \mathrm{~g}$, the actual stress condition in the prototype scale cannot be replicated, and hence, the dilatancy behaviour of soil cannot be properly captured. Liu et al. [9] carried out three-dimensional finite element analysis to study the effects of tunnelling on the existing support system in rock mass when a new tunnel was constructed perpendicularly above and beneath existing tunnels, i.e., effects of construction sequence on the existing tunnel. The computed results revealed that the shotcrete lining experienced tension in the spring line, whereas the crown and the invert experienced compression for both construction sequences. Attention was mainly given to the structural components, i.e., responses of rock bolts and shotcrete lining. Also, stress transfer mechanism in the soil nearby the existing tunnel was not studied. 
Until now, the effects of construction sequences for perpendicularly crossing tunnels constructed in sand have not been studied previously. It is well recognised that different construction sequences of tunnels result in different stress changes in the ground and hence induce different stress transfer mechanisms (i.e., stress redistribution) in the soil. This leads to different ground surface settlements and deformations, and hence, different bending moment induced on tunnel lining. Therefore, it is necessary to investigate the effects of construction sequences on the nearby existing tunnel. In this study, a series of three-dimensional finite element analyses are conducted to investigate the effects of construction sequences on perpendicularly crossing tunnels and to compare those results with that of greenfield cases. The hypoplastic constitutive model for sand is adopted in the numerical analysis to consider the soil small-strain stiffness. Also, the computed results are compared with cases whereby tunnelling was simulated with only volume loss (i.e., without considering effect of weight loss) to demonstrate the significant influence of modelling weight loss on different construction sequences. The induced ground surface settlements, deformation and displacement of the existing tunnel, bending moment and axial forces induced on the existing tunnel are reported and discussed. The stress transfer mechanism is also discussed and used to observe the behaviour of soil in this study.

\section{Numerical Model}

2.1. Finite Element Mesh and Boundary Conditions. Figures 1 and 2 show the plan view and the elevation view of the problem analysed for both cases S1 and S2. Tunnelling sequence $S 1$ refers to the case of a new tunnel constructed above an existing tunnel, while tunnelling sequence S2 refers to the case of a new tunnel constructed beneath an existing tunnel. The length and diameter of the both new and existing tunnels are assumed to be $100 \mathrm{~m}$ and $5 \mathrm{~m}$, respectively, with a tunnel lining thickness of $0.2 \mathrm{~m}$. According to Chinese specification (Specifications for Design of Highway Tunnels, 2014), the minimum thickness of tunnel lining is prescribed as $0.2 \mathrm{~m}$ for sandy soil. Thus, the thickness of the tunnel lining in this study is assumed to be $0.2 \mathrm{~m}$. The pillar width-to-diameter ratio, $P / D$, is 1.0 where the pillar width is defined as the shortest clearance distance between the outer tunnel linings. The cover-to-diameter ratio, $C / D$, is assumed to be 8.5 , i.e., $42.5 \mathrm{~m}$ from the ground surface. The finite element program ABAQUS [10] is used to model the three-dimensional tunnel excavation above and beneath the existing tunnel.

Figure 3 shows the three-dimensional finite element mesh for case S1, where the new tunnel axis is located at a depth of $45 \mathrm{~m}$ below the ground surface and $10 \mathrm{~m}$ above the existing tunnel axis. The mesh for case S2 is similar to that for case S1, and the locations of the new tunnel and the existing tunnel are just the opposite to that in S1. In addition, extra two cases of the greenfield condition (corresponding to S1 and S2) were analysed, in order to compare the ground surface settlement trough of S1 and S2 with that of the greenfield case. The meshes of two greenfield cases are the same as those of S1 and S2, respectively, but excluding the

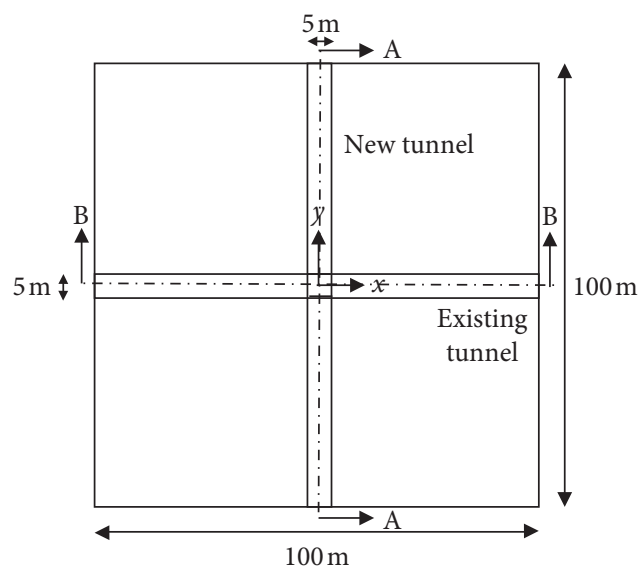

Figure 1: Plan view of S1 and S2.

existing tunnel. The six cases are summarised in Table 1 . The mesh has a dimension of $100 \mathrm{~m}$ (length) $\times 100 \mathrm{~m}$ (width) $\times$ $100 \mathrm{~m}$ (height) and consists of 22556 elements and 26341 nodes. A four-node shell element is used to model the tunnel lining, whereas an eight-node brick element is used to model the soil. The boundary conditions adopted for the finite element analysis include pin supports applied to the base and 4 vertical sides of the mesh. This restrains the movement in any directions at the base and vertical sides of the mesh.

2.2. Constitutive Model and Model Parameters. The hypoplastic (HP) model was developed to describe nonlinear response of granular materials [11]. The model is formulated by a single nonlinear tensorial equation, which establishes the relationship between the stress rate tensor $(\dot{\sigma})$ and the strain rate tensor $(\dot{\varepsilon})$, as follows:

$$
\dot{\sigma}=L(\sigma, e): \dot{\varepsilon}+N(\sigma, e): \dot{\varepsilon}
$$

In the first half of the equation (i.e., $L(\sigma, e): \dot{\varepsilon}$ ), the stress rate increases linearly with respect to the strain rate, while in the second half (i.e., $N(\sigma, e): \dot{\varepsilon}$ ) of the equation, a nonlinear relationship between the stress rate and the strain rate is established. $L(\sigma, e)$ and $N(\sigma, e)$ are $4^{\text {th }}$ and $2^{\text {nd }}$ constitutive tensors, respectively. $\dot{\varepsilon}: \dot{\varepsilon}$ is the Euclidean norm of the strain rate tensor, i.e., $\dot{\varepsilon}: \dot{\varepsilon}=\sqrt{\operatorname{tr}(\dot{\varepsilon})^{2}}$. The constitutive tensors $L$ and $N$ are both functions of soil states (i.e., effective mean normal stress $p^{\prime}$ and void ratio $e$ ). In the $e$ - $p^{\prime}$ space, three limiting boundaries are defined, as shown in Figure 4 . The pressuredependent void ratios follow the relationship below:

$$
\frac{e_{i}}{e_{i o}}=\frac{e_{c}}{e_{c o}}=\frac{e_{d}}{e_{d o}}=\exp \left(\frac{p^{\prime}}{h_{s}}\right)^{n},
$$

where $h_{s}$ and $n$ are the two parameters used for curve fitting and ei, ed, and ec are referred to the maximum void ratio (under isotropic compression), the minimum void ratio, and the critical state void ratio, respectively, while eio, edo, and eco denote the maximum, the minimum, and the critical state void ratios at zero confining stress, respectively.

The constitutive tensor $L$ in equation (1) is expressed as follows: 


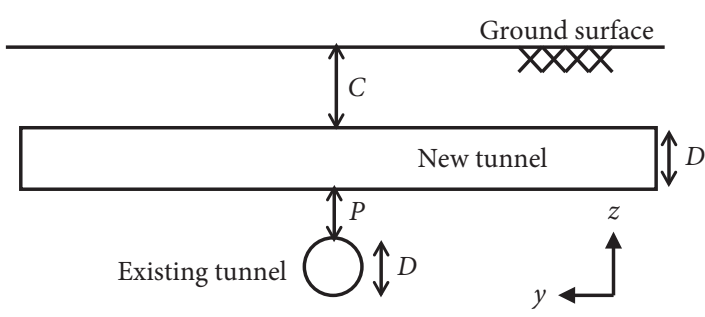

(a)

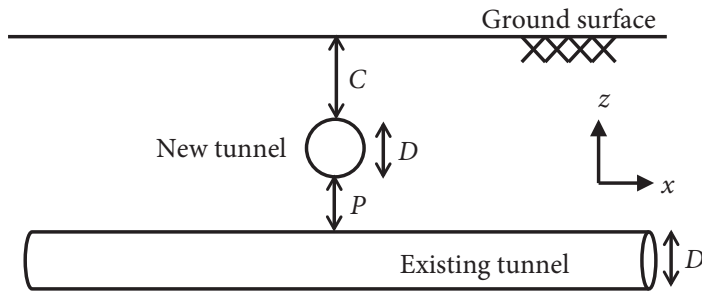

(c)

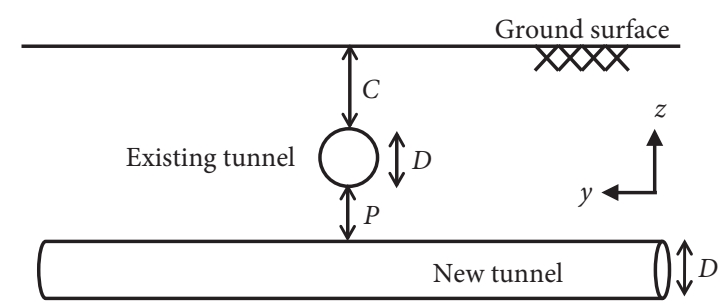

(b)

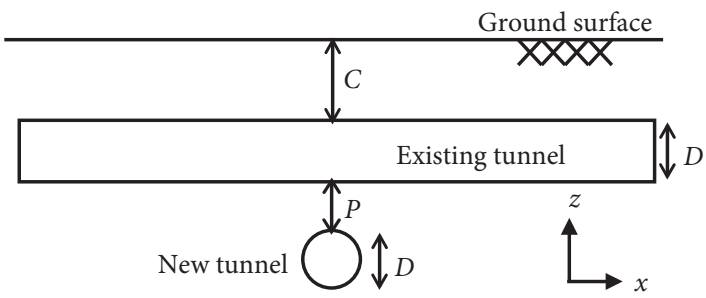

(d)

Figure 2: Elevation views of S1 and S2 (schematic diagram). (a) Tunnelling sequence S1 (Section A-A). (b) Tunnelling sequence S2 (Section A-A). (c) Tunnelling sequence S1 (Section B-B). (d) Tunnelling sequence S2 (Section B-B).

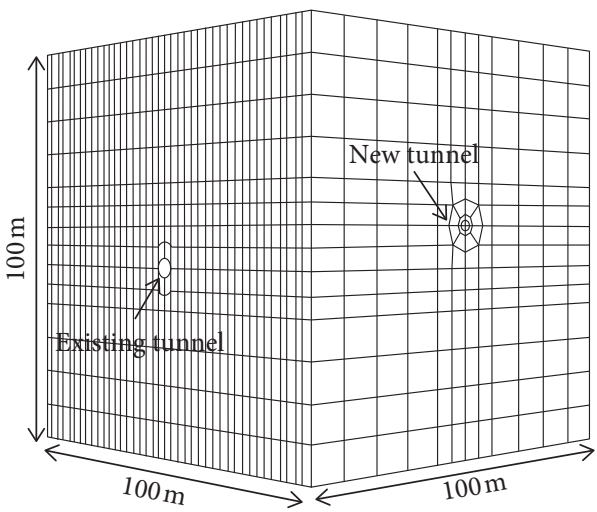

Figure 3: Finite element mesh for S1.

TABle 1: Numerical analysis program.

\begin{tabular}{|c|c|c|c|}
\hline Case & Description & $\begin{array}{l}C / D \text { new } \\
\text { tunnel }\end{array}$ & $\begin{array}{c}C / D \text { existing } \\
\text { tunnel }\end{array}$ \\
\hline S1-gf & $\begin{array}{l}\text { S1 (greenfield, i.e., without } \\
\text { existing tunnel) }\end{array}$ & 8.5 & N/A \\
\hline S2-gf & $\begin{array}{l}\text { S2 (greenfield, i.e., without } \\
\text { existing tunnel) }\end{array}$ & 10.5 & N/A \\
\hline S1-vl & $\begin{array}{l}\text { S1 (only volume loss, i.e., } \\
\text { without weight loss) }\end{array}$ & 8.5 & 10.5 \\
\hline S2-vl & $\begin{array}{l}\text { S2 (only volume loss, i.e., } \\
\text { without weight loss) }\end{array}$ & 10.5 & 8.5 \\
\hline S1 & $\begin{array}{c}\text { New tunnel above existing } \\
\text { tunnel }\end{array}$ & 8.5 & 10.5 \\
\hline S2 & $\begin{array}{l}\text { New tunnel beneath existing } \\
\text { tunnel }\end{array}$ & 10.5 & 8.5 \\
\hline
\end{tabular}

$$
L=f_{e} f_{b}(a I+\widehat{\sigma} \otimes \widehat{\sigma})
$$

where $I$ denotes the $4^{\text {th }}$-order unit tensor. $\widehat{\sigma}$ is the stress ratio tensor, being equal to $\sigma / \operatorname{tr} \sigma$. The parameter $a$ is a

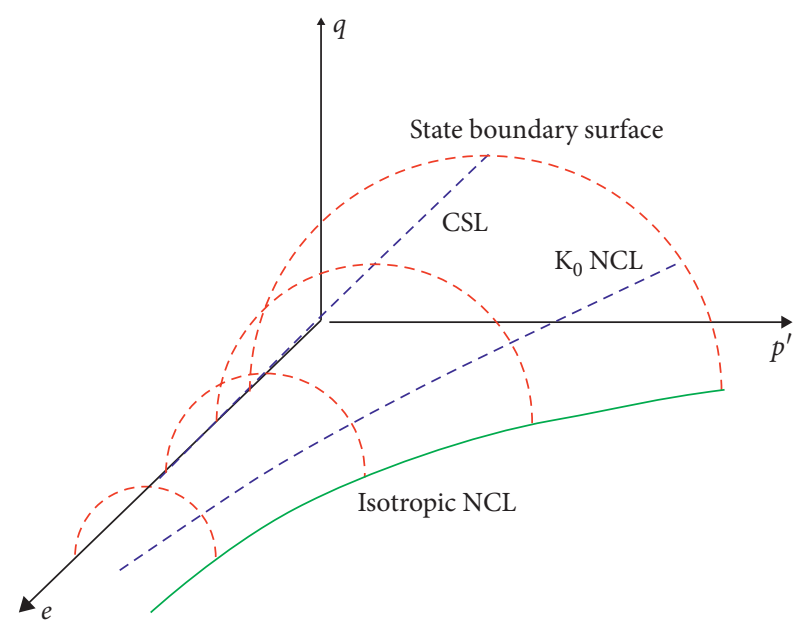

Figure 4: Illustration of state boundary surface in the hypoplastic model.

material constant depending on the effective friction angle at critical state $\left(\phi_{c}^{\prime}\right)$, as formulated in equation (4), while the scalars $f_{e}$ and $f_{b}$ are expressed by equations (5) and (6), respectively:

$$
\begin{aligned}
a= & \frac{\sqrt{3}\left(3-\sin \phi_{c}^{\prime}\right)}{2 \sqrt{2} \sin \phi_{c}^{\prime}}, \\
f_{e}= & \left(\frac{e_{c}}{e}\right)^{\beta}, \\
f_{b}= & \frac{h_{s}}{n} \frac{1+e_{i}}{e_{i}}(1.2)^{\beta}\left(\frac{3 p^{\prime}}{h_{s}}\right)^{1-n}\left(\frac{1}{c_{1}^{2}}\right) \\
& \cdot\left[1+\frac{c_{1}^{2}}{3}-\left(\frac{1.2-e_{i o} / e_{c o}}{1-e_{i o} / e_{c o}}\right)^{\alpha} \frac{c_{1}}{\sqrt{3}}\right]^{-1} .
\end{aligned}
$$


The parameter $\alpha$ is related to soil dilation, while $\beta$ controls soil stiffness at relatively large strain levels (shear strain $>1 \%$ ). To be more specific, an increasing value of $\alpha$ leads to a more dilative response of the soil. A larger $\beta$ is associated with a stiffer soil response at relatively large strains. The parameter $c_{1}$ is a function of $\phi_{c}^{\prime}$, expressed as follows:

$$
c_{1}=\sqrt{\frac{3}{8}} \frac{\left(3-\sin \phi_{c}^{\prime}\right)}{\sin \phi_{c}^{\prime}} .
$$

In addition to $L$, the other constitutive tensor $N$ in equation (1) is formulated as follows:

$$
N=f_{e} f_{b} f_{d}(\widehat{\sigma}+\widehat{s}),
$$

where $\widehat{s}$ denotes the deviatoric stress tensor, being equal to $\bar{\sigma}-(1 / 3) I$. The scalar $f_{d}$ is a function of $e_{c}, e_{d}$, and current void ratio $(e)$, expressed by the following equation:

$$
f_{d}=\left(\frac{e-e_{d}}{e_{c}-e_{d}}\right)^{\alpha} .
$$

In summary, the basic hypoplastic model consists of eight model parameters, namely, $\phi_{c}^{\prime}, h_{s}, n, e_{i o}, e_{d o}, e_{c o}, \alpha$, and $\beta$.

To account for strain dependency and path dependency of soil stiffness at small strains, Niemunis and Herle [12] further improved the basic HP model by incorporating intergranular strain concept into the model. Five additional parameters (i.e., $m_{R}, m_{T}, R, \beta_{r}$, and $\chi$ ) are required. Among the five parameters, $\mathrm{m}_{R}$ and $\mathrm{m}_{T}$ control the initial shear modulus upon $180^{\circ}$ and $90^{\circ}$ strain path reversal, respectively. $R$ denotes the elastic strain range. $\beta_{r}$ and $\chi$ are used to define the degradation rate of stiffness with strain.

In this study, the geotechnical profile is assumed to be Toyoura sand which is widely used in previous research to investigate sand properties. The parameters of Toyoura sand for hypoplastic model summarized in Table 2 are obtained from Hong et al. [13], who predicted soil deformation in a centrifuge experiment on tunnel excavation with hypoplastic model.

The concrete tunnel lining was modelled as a linear elastic material with Young's modulus of $30 \mathrm{GPa}$ and Poisson's ratio of 0.25 [14]. The effective unit weight for the tunnel lining was $25 \mathrm{kN} / \mathrm{m}^{3}$ which is a widely adopted value for unit concrete weight [15].

2.3. Numerical Modelling Procedures. A total of six threedimensional analyses are carried out as summarised in Table 1. Among the six analyses, two analyses with different construction sequences, S1 and S2, are carried out to study the effects of newly constructed tunnel on the response of the ground and the existing tunnel. To study the influence of the existing tunnel (providing stiffening effect to the ground) on the ground surface settlement due to the newly constructed tunnel, two additional greenfield cases (corresponding to S1 and S2) are also studied. Another two analyses are carried out where tunnelling is simulated with only volume loss (i.e., without considering effect of weight loss) to demonstrate the
TABLE 2: Soil parameters used in finite element analyses.

\begin{tabular}{lc}
\hline Description & Parameter \\
\hline $\begin{array}{l}\text { Effective angle of shearing resistance at critical state: } \\
\phi^{\prime}\end{array}$ & $31^{\circ}$ \\
Coefficient of at rest earth pressure, $K_{o}$ & 0.5 \\
Hardness of granulates, $h_{s}$ & $2.6 \mathrm{GPa}$ \\
Exponent $n$ & 0.27 \\
Minimum void ratio at zero pressure, $e_{d o}$ & 0.61 \\
Maximum void ratio at zero pressure, $e_{i o}$ & 1.10 \\
Critical void ratio at zero pressure, $e_{c o}$ & 0.98 \\
Exponent $\alpha$ & 0.11 \\
Exponent $\beta$ & 4 \\
Parameter controlling the initial shear modulus upon & 8 \\
$180^{\circ}$ strain path reversal, $m_{R}$ & \\
Parameter controlling the initial shear modulus upon & 4 \\
90 strain path reversal, $m_{T}$ & $2 \times 10^{-5}$ \\
Size of elastic range, $R$ & \\
Parameter controlling the degradation rate of & 0.15 \\
stiffness with strain, $\beta_{r}$ & \\
Parameter controlling the degradation rate of & 1.0 \\
stiffness with strain, $\chi$ &
\end{tabular}

significant influence of modelling weight loss on different construction sequences. In other words, soil is excavated surrounding the tunnel lining only to simulate volume loss of $2 \%$ without excavating the soil inside the tunnel lining. Based on the data of ground loss ratio induced by shield tunnel construction in Beijing, Shanghai, Nanjing, Guangzhou, Wuhan, Tianjin, and Shenzhen, seventy-one groups of data are statistically analysed. The results show that the ground loss ratio ranges from $0.2 \%$ to $3.01 \%$ [16]. Therefore, the volume loss of $2 \%$ assumed in this study is reasonable. The meshes of two greenfield cases are the same as those of S1 and S2, respectively, but excluding the existing tunnel. The numerical model adopted in this study for simulating tunnel excavation nearby a perpendicularly crossing existing tunnel has been widely used and validated by previous research [17-19]. For instance, $\mathrm{Ng}$ et al. [18] conducted three-dimensional numerical analyses to investigate the effect of tunnel construction on overlying existing perpendicularly crossing tunnel based on a centrifuge experiment, in which the geometric size and modelling strategy for simulating tunnel excavation have significant similarity with that of this study. The high consistency between the computed results and experimental data in the study of $\mathrm{Ng}$ et al. [18] could provide a solid validation for the numerical model adopted in this study.

Figure 5 illustrates the procedure of the tunnel excavation adopted in the analysis. In this analysis, the existing tunnel is modelled as wished-in-place. The new tunnel (100 $\mathrm{m}$ long) is divided into 80 sections whereby $1.25 \mathrm{~m}$ per section of soil is excavated in each excavation stage. Tunnel excavation is simulated by removing the soil located in the excavation zone; i.e., soil is excavated inside the tunnel lining and surrounding the tunnel lining by deactivating the soil element. As for the cases consider only volume loss without the effect of weight loss, soil is excavated surrounding the tunnel lining only. Before excavation of the new tunnel, the lining is placed inside the new tunnel without activating it. 


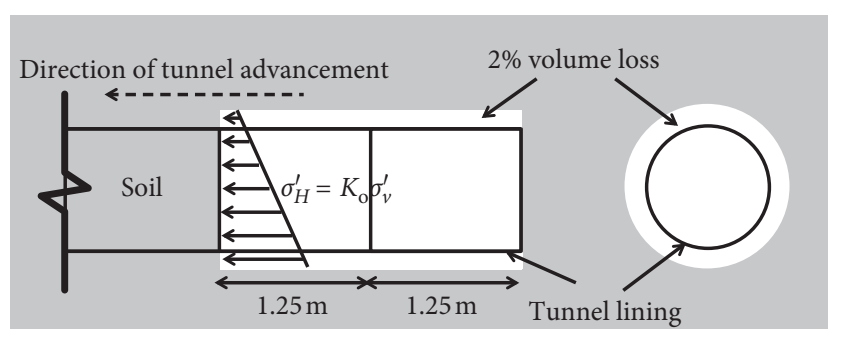

(a)

(b)

FIGURE 5: Schematic diagram of numerical modelling procedure. (a) Longitudinal view. (b) Cross-sectional view.

Therefore, there is no physical interaction between the soil and the tunnel lining, prior to tunnel advancement. The lining of the new tunnel is activated and interaction between the soil and the lining occurs when the soil deforms due to excavation and comes into contact with the surface of the tunnel lining. As shown Figure 4, the gap between the soil and the outer diameter of the tunnel is equivalent to a $2 \%$ volume loss. In this way, there is no unsupported length during tunnelling but a simplified way of displacement control for $2 \%$ volume loss surrounding the tunnel lining. The tunnel excavation procedure can be regarded as shield tunnelling. This modelling technique allows simulation of both weight and volume losses during tunnelling. No horizontal displacement is allowed at the tunnel face during each tunnel advancing step. The tunnel excavation modelling strategy is also adopted by Zhao et al. [20] and Boonyarak and Ng [19], who conducted the tunnel excavation experiment and compared the measured data with the numerical results. The considerable consistency between the measured data and the numerical results proved that the tunnel excavation modelling strategy in this study can be used to correctly estimate the soil and lining deformations for this problem. Figure 6 shows a schematic diagram for the modelling procedure.

\section{Computed Results}

3.1. Ground Surface Settlement. Figure 7 shows the computed transverse surface settlement troughs along section B-B at the end of excavation for all six cases. For the twin tunnel cases (both S1 and S2), the maximum ground surface settlement for case S1 is larger than that for case S2 whereby the maximum ground settlements for cases $\mathrm{S} 1$ and $\mathrm{S} 2$ are $6.6 \mathrm{~mm}$ and $6.2 \mathrm{~mm}$, respectively. This is because the $C / D$ ratio for the new tunnel for case $\mathrm{S} 1$ is smaller than that for case $\mathrm{S} 2$. The induced ground settlement troughs under the greenfield condition are also included in this figure for comparison (i.e., cases S1-gf and S2-gf). The maximum ground surface settlements under greenfield conditions (i.e., cases S1-gf and S2-gf) are larger as compared to those of corresponding twin tunnel cases (i.e., cases S1 and S2), respectively. This is due to the presence of the existing tunnel which stiffens the soil, resulting in an approximately $14 \%$ reduction of the maximum ground surface settlement for both cases S1 and S2.

On the contrary, the ground surface settlements for cases S1-vl and S2-vl are smaller than those of both twin tunnel and greenfield cases. The maximum ground surface

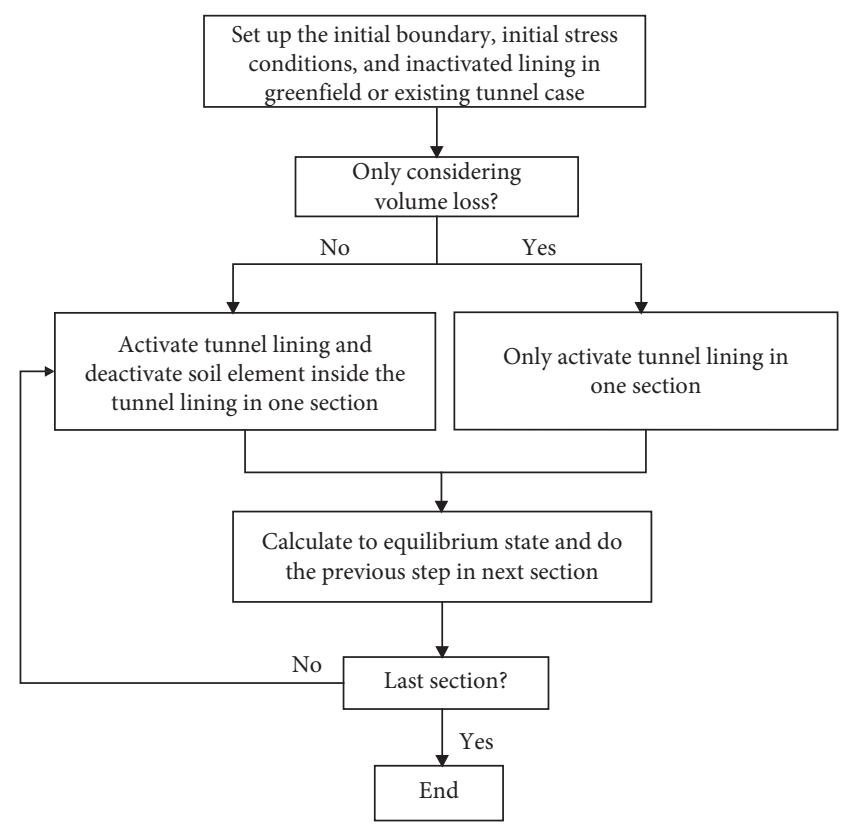

FIGURE 6: Schematic diagram for the modelling procedure.

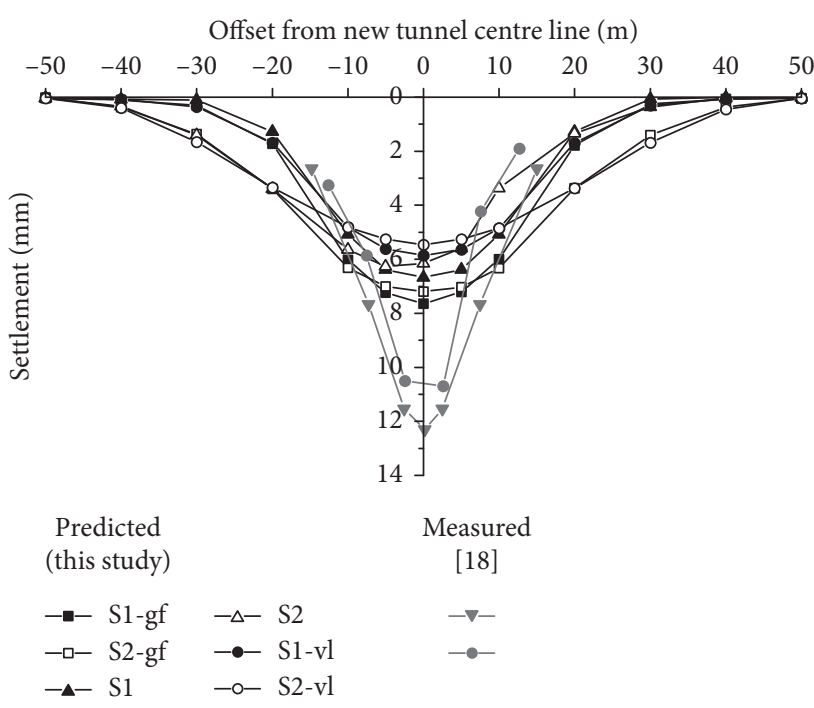

FIgURE 7: Comparison of transverse ground surface settlement troughs.

settlements for cases S1-vl and S2-vl are $5.8 \mathrm{~mm}$ and $5.4 \mathrm{~mm}$, respectively. In other words, the maximum ground surface settlements for cases S1-vl and S2-vl are 12\% and 13\% smaller than those for cases S1 and S2, respectively. It can be explained that removal of soil weight inside the tunnel lining causes changes in total stress around the new tunnel. Subsequently, larger stress relief occurs around the new tunnel for cases S1 and S2 as compared to those for S1-vl and S2-vl which only consider the effect of volume loss without the effect of weight loss. Therefore, the ground surface settlements are smaller than those for cases S1 and S2.

3.2. Longitudinal Tunnel Settlement. Figure 8 shows the distribution of longitudinal settlement of the existing 
Offset from existing tunnel center line (m)

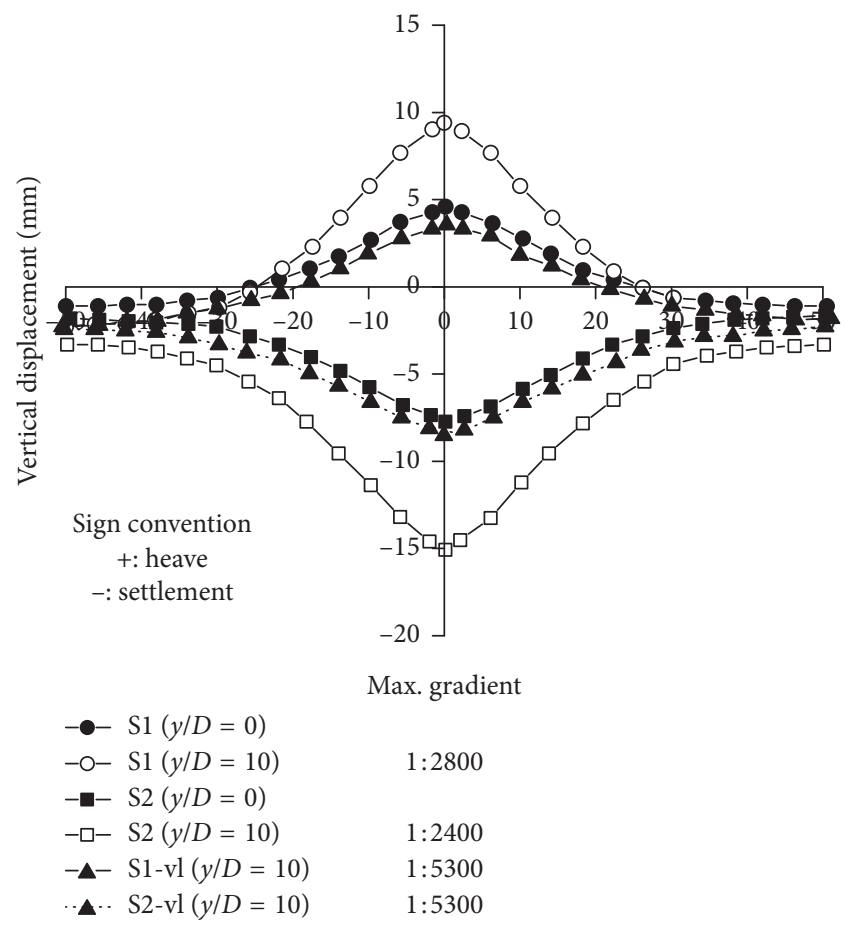

FIGURE 8: Comparison of longitudinal tunnel vertical displacement.

tunnel along section B-B (Figure 1) for different advancing stages expressed in terms of $y / D$ ratios. Each $y / D$ ratio represents the normalised advanced distance of the tunnel face with the diameter of the tunnel. For instance, at $y /$ $D=-10$, the tunnel face is at a distance of $10 D$ behind the existing tunnel centre line (i.e., monitoring section B-B). The tunnel is assumed to be continuous without joints (i.e., tunnel lining is not segmented). The middle section of the existing tunnel displaces upward (i.e., heave) for cases $\mathrm{S} 1$ and S1-vl and downward (i.e., settle) for cases S2 and S2-vl as the $y / D$ ratio increases. This is because tunnel excavation results in stress relief on each existing tunnel hence causing each existing tunnel to move towards each new tunnel. The differential settlement and changes of gradient of the existing tunnel due to the construction of the new tunnel is a concern for tunnel design and shall be addressed in this section. The maximum heave in case S1 and maximum settlement in case S2 of the existing tunnel are $9 \mathrm{~mm}$ and $16 \mathrm{~mm}$, respectively. The differential settlements of the existing tunnel for cases $\mathrm{S} 1$ and $\mathrm{S} 2$ are $11.6 \mathrm{~mm}$ and $11.8 \mathrm{~mm}$, respectively. The differential settlement is equivalent to a gradient of $1: 2800$ and $1: 2400$ for cases S1 and S2, respectively. The differential settlements and changes in gradient for case S2 is slightly larger than that for case S1 due to larger effect of stress relief which are discussed later. On the contrary, the differential settlements for cases S1-vl and S2-vl are smaller than those for cases S1 and S2. The differential settlements for cases S1-vl and S2-vl are 54\% and 53\% smaller than those for cases S1 and S2, respectively. This is due to the absence of weight loss which results in smaller effect of stress relief for cases S1-vl and S2-vl as compared to that for cases S1 and S2.
3.3. Displacement and Deformation of Existing Tunnel. Figure 9 shows the displacement and deformed shape of the existing tunnel at section A-A for different advancing stages expressed in terms of $y / D$ ratios. The existing tunnel displaces upward (i.e., heave) for case S1 and downward (i.e., settle) for case $\mathrm{S} 2$ as the $y / D$ ratio increases. This is because tunnel excavation results in stress relief on each existing tunnel hence causing each existing tunnel to move towards each new tunnel. The maximum upward and downward vertical displacements of the existing tunnel for cases $\mathrm{S} 1$ and S2 are approximately $9.4 \mathrm{~mm}$ and $15.0 \mathrm{~mm}$, respectively, at the end of excavation (i.e., at $y / D=10$ ). In other words, the maximum downward vertical displacement for case S2 is $60 \%$ larger than the upward displacement for case S1. This is because the upward movement (i.e., heave) of the tunnel for case $S 1$ is hindered by the overburden pressure of the soil above the existing tunnel which leads to smaller upward displacement of the existing tunnel (Figure 8). On the contrary, downward displacement for case S2 is caused by both stress reduction at the invert and the overburden pressure of soil above the existing tunnel. When the tunnel face is at $y / D=-1$, the existing tunnel displaces towards the new tunnel face and displaces back to its original position in the horizontal direction after the tunnel face passes the monitoring section. The existing tunnels tended to displace towards the advancing tunnel face in the $y$ direction during tunnelling due to the effect of stress relief.

In addition to displacement of the existing tunnel, deformation of the existing tunnel is also observed. The horizontal diameter decreases (i.e., compressed), but the vertical diameter increases (i.e., elongates) with increasing $y /$ $D$ ratio due to the effect of stress relief for both cases S1 and S2. The deformation of existing tunnels for case S1 is slightly smaller than that for case S2 in terms of both elongation and compression. The vertical elongation and horizontal compression of the existing tunnel for case S2 are 6\% and 9\% larger than those for case S1, respectively. The vertical elongation of each existing tunnel is mainly due to the deformation of the tunnel lining at the crown for case S1 and the invert for case S2. This is further verified by considering the change of normal stresses around the tunnel leading to bending moment induced on the tunnel lining, which are discussed later. The magnitudes of vertical elongation are approximately $33 \%$ and $54 \%$ larger than the horizontal compression for both cases S1 and S2, respectively, at the end of excavation (i.e., at $y / D=10$ ). This computed result is inconsistent compared with the physical model test result reported by Kim et al. [8]. Their experimental result shows an opposite phenomenon whereby the existing tunnel experienced a decrease in vertical diameter but an increase in horizontal diameter due to the construction of a new perpendicular tunnel. It is reported by the authors that this is because of the large jacking forces which tended to cause the horizontal diameter of adjacent tunnels to increase which counteract the expected tendency of the vertical diameter of the adjacent tunnels to increase as a result of stress relief [8]. On the contrary, the vertical displacement and deformation of each existing tunnel for cases S1-vl and S2-vl are smaller than those for cases S1 and S2. The upward and downward 


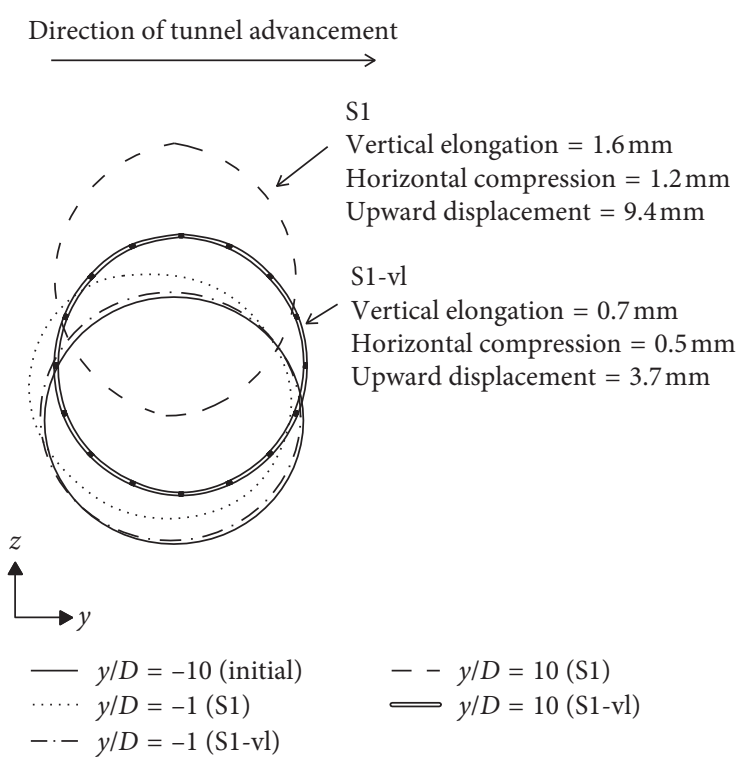

(a)

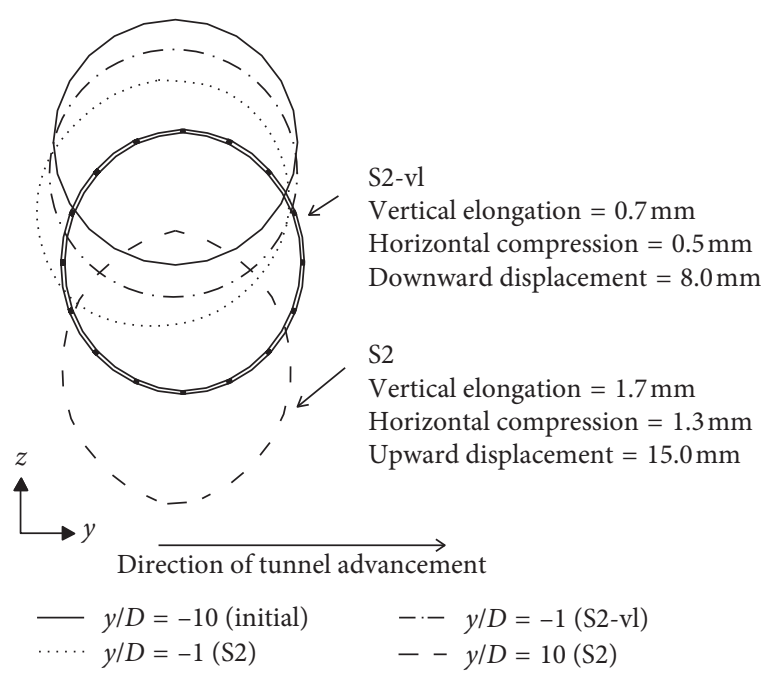

(b)

Figure 9: Displacement and deformation of the existing tunnel (not to scale) of (a) S1, case 1, and (b) S2, case 2.

vertical displacements for cases S1-vl and S2-vl are $61 \%$ and $47 \%$ smaller than those for cases S1 and S2, respectively. This is due to absence of weight loss which leads to smaller stress relief for cases S1-vl and S2-vl relief (Figure 10) as compared to those for cases S1 and S2. Similar to cases S1 and S2, the downward vertical displacement for case S2-vl is larger than the upward displacement for case S1-vl. This is because the effect of weight loss causes changes of total stress around the new tunnel. Subsequently, an additional stress reduction due to weight loss occurs on the existing tunnel when the new tunnel advances beyond the existing tunnel. The deformations of existing tunnel for cases S1-vl and S2-vl are smaller than those for cases S1 and S2, respectively. The magnitudes of vertical elongation for cases S1-vl and S2-vl are $56 \%$ and $59 \%$ smaller than those for cases S1 and S2, respectively. On the contrary, the magnitudes of horizontal compression for cases S1-vl and S2-vl are 58\% and 62\% smaller than those for cases S1 and S2, respectively. This is due to the absence of weight loss which leads to smaller stress relief (detail explanation is given in Section 3.6) for cases S1-vl and S2-vl as compared to those for cases S1 and S2. Similar to cases S1 and S2, the deformation for case S2-vl is larger than that of case S1-vl. This is because the magnitude of maximum bending moment at the crown for case S2-vl is larger than that of the invert for case S1-vl.

3.4. Bending Moment Induced on Existing Tunnel. Figures 11(a) and 11(b) show the distribution of transverse bending moment induced on the lining of the existing tunnel along section $\mathrm{A}-\mathrm{A}$ at the end of excavation. Positive bending moment denotes that the existing tunnel deformed outwards (i.e., outer face of tunnel lining in tension or hogging moment), while the negative bending moment denotes that the existing tunnel deformed inwards (i.e., outer face of tunnel lining in compression or sagging moment).
From Figures 11(a) and 11(b), the maximum (hogging) induced transverse bending moment occurs at the crown for cases S1 and S1-vl and at the invert of the existing tunnel for cases S2 and S2-vl, while the minimum (sagging) bending moment occurs at the spring line for all four cases S1, S2, S1$\mathrm{vl}$ and S2-vl. The elongation of the existing tunnel due to stress relief results in maximum positive (hogging) bending moment occurring at the crown and the invert for all the four cases. In contrast, the horizontal compression (Figure 9) of the existing tunnel results in minimum (sagging) bending moment occurring at the spring line (Figure 9). The magnitude of the maximum (hogging) and minimum (sagging) transverse bending moment in lining of the existing tunnel for case $\mathrm{S} 1$ is slightly smaller than that for case S2.

On the contrary, the maximum (hogging) induced transverse bending moments for cases S1-vl and S2-vl are smaller than those for cases S1 and S2, respectively. This is due to the absence of weight loss which leads to the smaller stress relief for cases S1-vl and S2-vl as compared to those for cases S1 and S2. The changes of stress are explained later in Section 3.6. Therefore, the vertical displacement and deformation of each existing tunnel for cases S1-vl and S2-vl are smaller than those for cases S1 and S2 (Figure 9). When comparing case S1-vl and case S2-vl, the maximum magnitude of hogging bending moment at the crown for case S2$\mathrm{vl}$ is larger than that of the invert for case S1-vl. Therefore, the downward vertical displacement for case S2-vl is larger than the upward displacement for case S1-vl (Figure 9). Figure 12 shows the distribution of longitudinal bending moment induced on the lining of the existing tunnel along section B-B (Figure 1) for different advancing stages expressed in terms of $y / D$ ratios. Each $y / D$ ratio represents the normalised advanced distance of the tunnel face with the diameter of the tunnel. The bending moment induced on existing tunnel lining are derived based on the longitudinal 


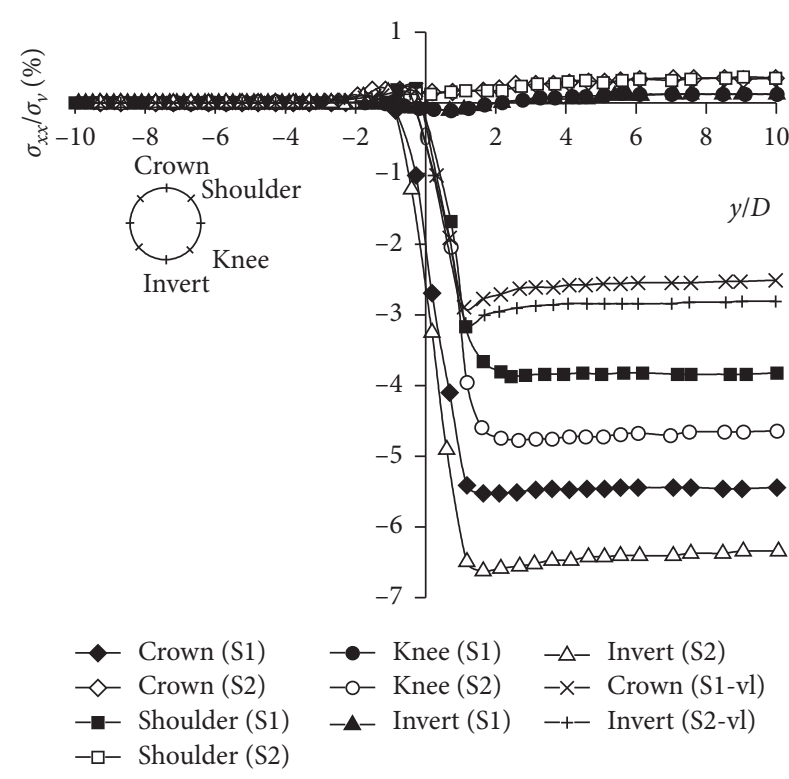

(a)

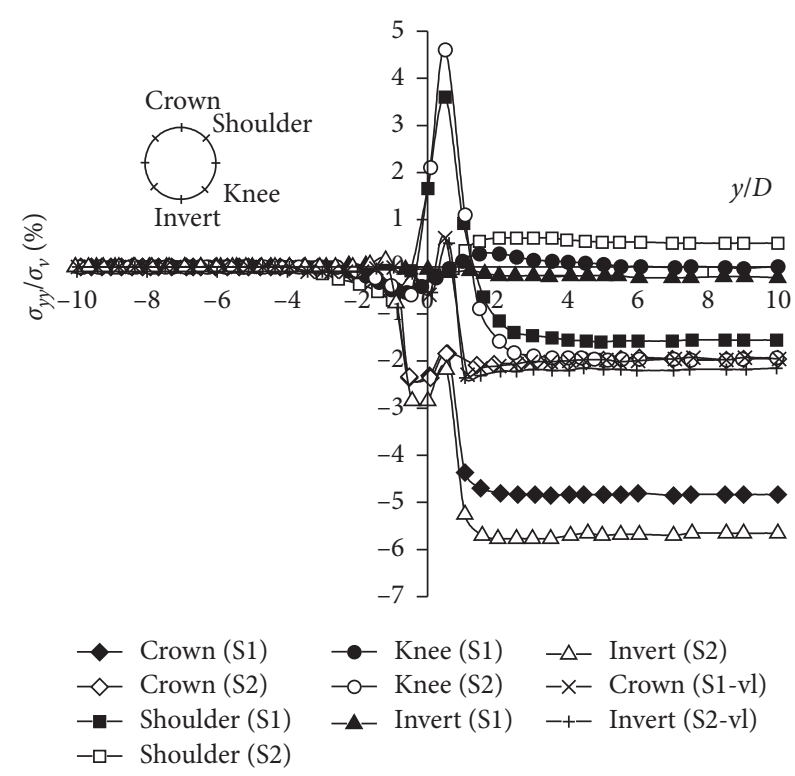

(b)

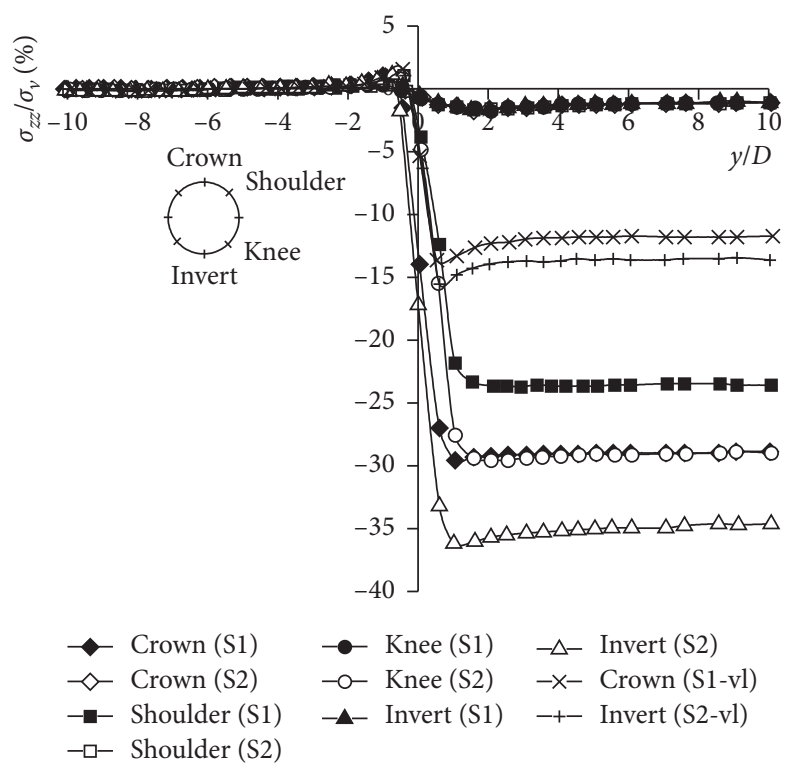

(c)

Figure 10: (a) Normalised longitudinal stress ratio, $\sigma_{x x} / \sigma_{v}$, (b) normalised transverse stress ratio, $\sigma_{y y} / \sigma_{v}$, and (c) normalised vertical stress ratio, $\sigma_{z z} / \sigma_{v}$, around the existing tunnel as tunnel face advances.

vertical displacement of the existing tunnel using the elastic beam theory stated below:

$$
M(x)=\frac{d^{2} \delta(x)}{d x^{2}} E_{p} I_{p}
$$

where $\delta(x)$ is the deformation of the existing tunnel lining, while $E_{p}$ and $I_{p}$ are Young's modulus and moment of inertia of the tunnel, respectively. The tunnel is assumed to be continuous without joints (i.e., tunnel lining is not segmented). As the tunnel face advances, the magnitudes of bending moment increase for both cases S1 and S2. The upward movement (i.e., heave) and downward movement (i.e., settle) of the existing tunnel for cases S1 and S2
(Figure 8) result in maximum positive (hogging) and maximum negative (sagging) bending moments occurred at the middle section of the existing tunnel, respectively. The magnitude of maximum (hogging) bending moment for case $\mathrm{S} 1$ is $19 \mathrm{MN} \cdot \mathrm{m}$ which is smaller than the sagging moment for case S2 $(20 \mathrm{MN} \cdot \mathrm{m})$. This is because the maximum reduction of the stress ratio for case $\mathrm{S} 1$ is smaller than that for case S2 which leads to a smaller vertical displacement and bending moment (Figures 9 and 11, respectively) for case S1 as compared to that for case S2. On the contrary, the maximum bending moments for cases S1$\mathrm{vl}$ and S2-vl are 47\% and 50\% smaller than those for cases S1 and S2, respectively. The smaller magnitude of bending 


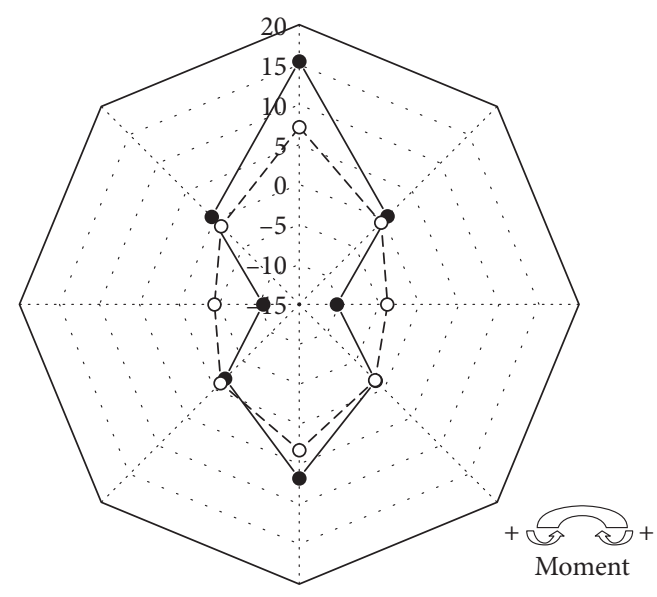

$-\mathrm{S} 1$

$-0-$ S1-vl

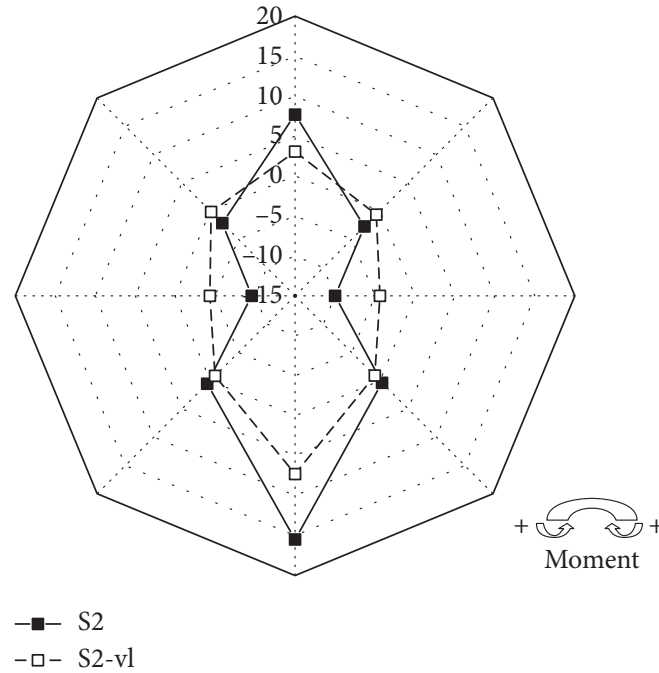

(b)

FIGURE 11: Transverse bending moments $(\mathrm{kN} \cdot \mathrm{m} / \mathrm{m})$ induced on the lining of the existing tunnel at the end of tunnel excavation of (a) S1 and (b) S2.

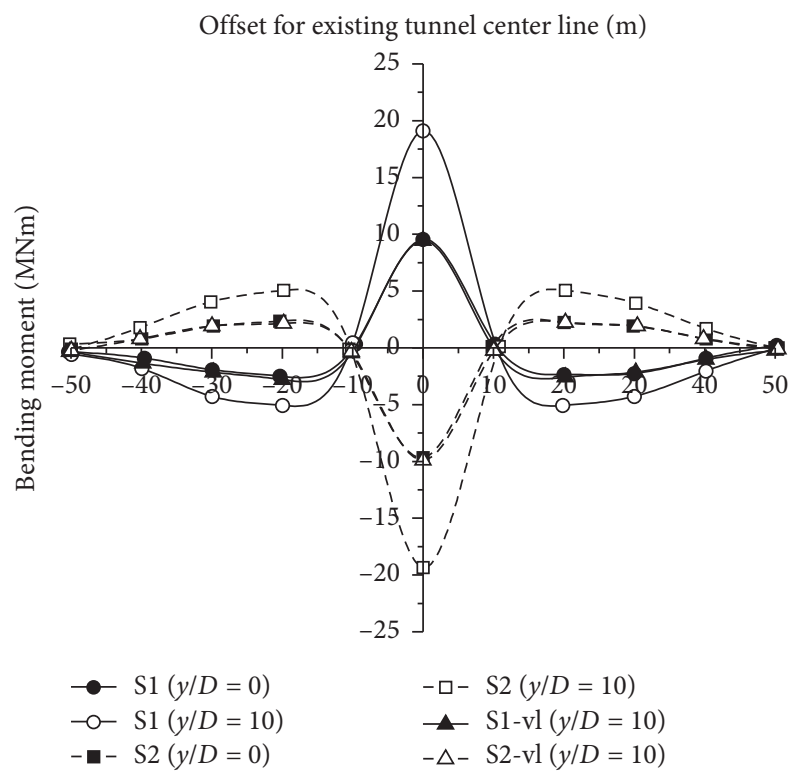

FIGURE 12: Longitudinal bending moment of the existing tunnel.

moment for cases S1-vl and S2-vl as compared to those for cases $\mathrm{S} 1$ and $\mathrm{S} 2$ is due to the smaller displacement of the existing tunnel for cases $\mathrm{S} 1-\mathrm{vl}$ and $\mathrm{S} 2$-vl as compared to those for cases $\mathrm{S} 1$ and $\mathrm{S} 2$. Also, the stress reduction for cases S1-vl and S2-vl is smaller than that for cases S1 and S2 (see Figure 10). When comparing case S1-vl and case S2-vl, the maximum bending moment for case $\mathrm{S} 1-\mathrm{vl}$ is similar to case S2-vl.

3.5. Axial Forces in the Existing Tunnel Lining. Figures 13(a) and 13(b) show the hoop force and longitudinal axial force in the existing tunnel lining along section $\mathrm{A}-\mathrm{A}$ at the end of excavation for cases S1, S1-vl, S2, and
S2-vl. In Figure 13(a), the maximum hoop force occurs at the spring line for both cases S1 and S2, while the minimum hoop force occurs at the crown for case S2 and the invert for case $\mathrm{S} 1$. The maximum and minimum axial forces are approximately $372 \mathrm{kN} / \mathrm{m}$ and $197 \mathrm{kNm} / \mathrm{m}$ for both cases $\mathrm{S} 1$ and S2, respectively. The hoop forces at the elements above the spring line are larger than those below the spring line for case S1 leading to upward movement, i.e., heave of the existing tunnel for case S1. On the contrary, the hoop forces at the elements below the spring line are larger than those above the spring line for case S2 leading to downward movement, i.e., settle of the existing tunnel for case S2. The trend of hoop force for case S1-vl and case S2-vl is similar to that for case S1 and case S2, respectively. However, the magnitudes of hoop force for case S1-vl and case S2-vl is smaller than those for case $S 1$ and case S2, respectively. This is due to the smaller deformation of the existing tunnel for cases S1-vl and S2-vl as compared to that for cases S1 and S2, respectively (Figure 9). Also, the stress reductions of cases S1-vl and S2-vl are smaller than those of cases S1 and S2, respectively (Figure 10).

From Figure 13(b), the maximum longitudinal axial force occurs at the crown for case $\mathrm{S} 1$ and at the invert for case S2, while the minimum axial force occurs at the invert for case S1 and at the crown for case S2. This is due to the significant effect of stress relief near the crown for case S1 and at the invert for case S2. The largest magnitudes of axial forces experienced in the lining for cases S1 and S2 are approximately $579 \mathrm{kN} / \mathrm{m}$ and $584 \mathrm{kN} / \mathrm{m}$, respectively. The trend of axial force for case S1-vl and case S2-vl is similar to that for case S1 and case S2, respectively. However, the magnitude of axial force for case S1-vl and case S2-vl is smaller than that for case S1 and case S2, respectively. This is due to the smaller deformation of the existing tunnel for cases S1-vl and S2-vl as compared to that for cases S1 and S2, respectively (Figure 9). Also, the stress reduction of cases 


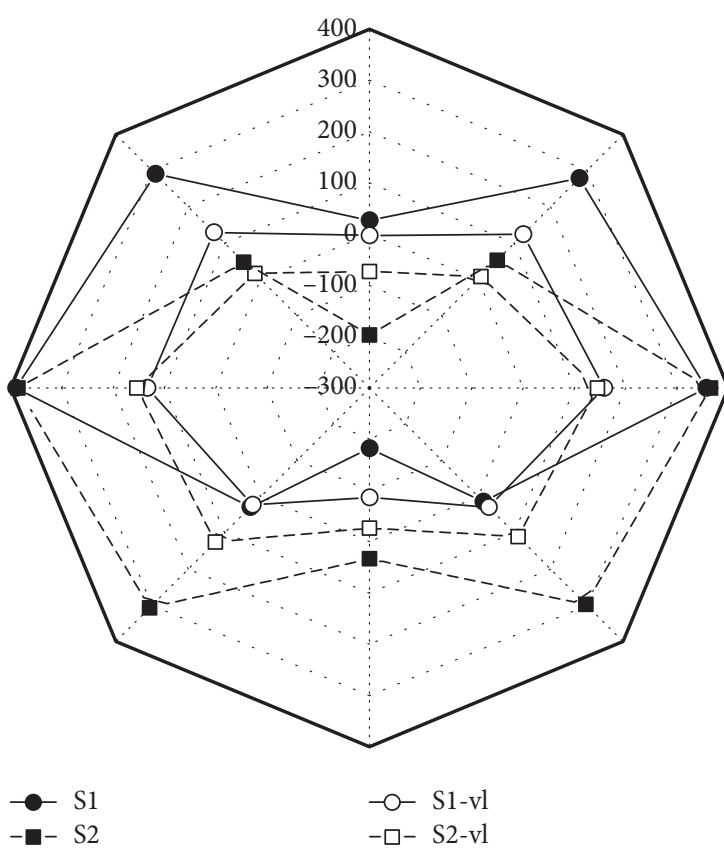

(a)

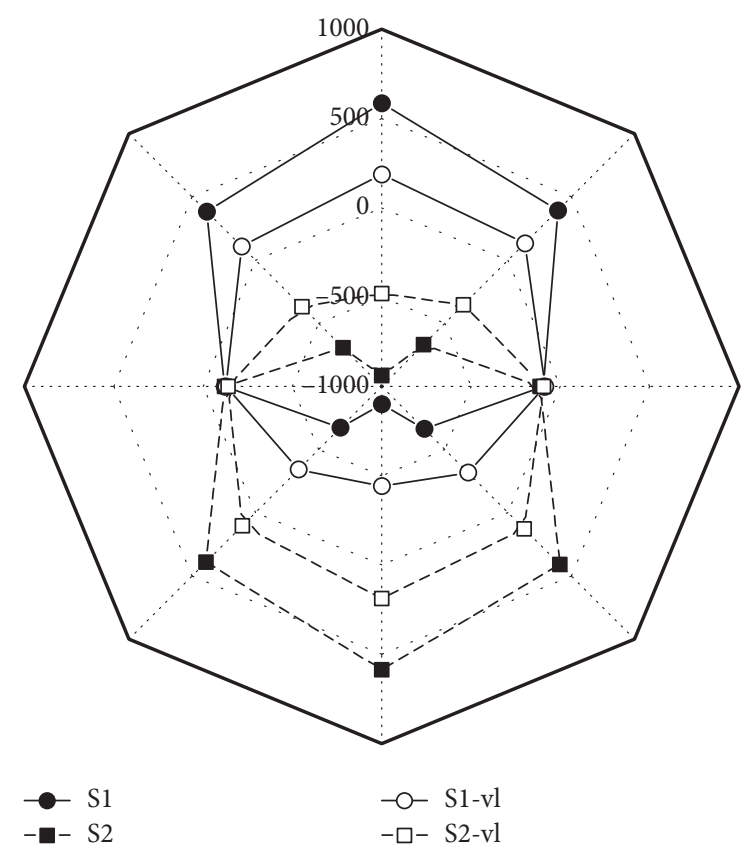

(b)

Figure 13: (a) Hoop force and (b) longitudinal axial force $(\mathrm{kN} / \mathrm{m})$ in the lining of the existing tunnel at the end of tunnel excavation of $\mathrm{S} 1$ and $\mathrm{S} 2$.

S1-vl and S2-vl is smaller than that of cases S1 and S2, respectively.

3.6. Stress Transfer Mechanism. Figure 10 shows the normal stresses in soil which include longitudinal ( $x$ direction), transverse ( $y$ direction), and vertical ( $z$ direction) stresses around the existing tunnel at different tunnel advancing stages along section A-A. A total of four soil elements which are located at the crown, shoulder, knee, and invert at the monitoring section are chosen for both cases S1 and S2. Also, the soil elements at the crown for S1-vl and the invert for S2-vl are included in these figures for comparison. For ease of comparison, the normal stresses are normalised by the corresponding overburden pressures and are expressed in terms of percentage. Also, the tunnel advancing stages are expressed in terms of normalised distance of the advancing tunnel face with the diameter of the tunnel, $y / D$.

Figure 10(a) shows that the normalised stress ratio in the longitudinal direction, $\sigma_{x x} / \sigma_{v}$, increases slightly when the tunnel face reaches $y / D=-3$. However, as the tunnel face approaches the centre line of the existing tunnel, the stress ratios at the crown and shoulder for case S1 and the knee and invert for case S2 decrease remarkably. The stress ratios reach their minimum values when the tunnel face arrives at $y /$ $D=1.5$. The stress ratios at the crown for case $\mathrm{S} 1$ and the invert for case S2 then increase (i.e., recover) slightly to different extent as a result of stress transfer in the longitudinal direction of the existing tunnel when the tunnel face reaches $y / D=2$, beyond which no further stress changes take place.

In Figure 10(b), noticeable normal stress changes take place in the transverse direction (i.e., a slight increase in stress) when the tunnel face reaches $y / D=-3$. The normalised transverse stress ratios, $\sigma_{y y} / \sigma_{v}$, for soil elements at the shoulder for case S1 and the knee for case S2 increase tremendously when the tunnel face arrives at $y / D=-0.5$ and reaches its maximum at $y / D=0.5$. After reaching its maximum, it begins to decrease and remains constant after $y /$ $D=2$. The increase in stress ratios at the shoulder for case $S 1$ and the knee for case S2 and the decrease of the stress ratios at the crown for case S1 and the invert for case S2 indicate that the stresses from the crown of case S1 and the invert of case S2 are transferred to the shoulder of case S1 and the knee of case S2. A slight increase in the normalised transverse stress ratios at the knee of case S1 and the shoulder of case $\mathrm{S} 2$ is also observed at $y / D=0$, starts to decrease (i.e., recover) at $y / D=2$, and remains constant after $y / D=6$.

\section{Conclusions and Limitations}

Based on the computed results of the three-dimensional finite element analyses, the following conclusions are drawn:

(1) The ground surface settlement for case S1 is deeper but narrower as compared to that for case S2. The computed results for both cases S1 and S2 show a smaller maximum settlement as compared to that of the greenfield case. This is due to the presence of existing tunnel beneath and above the new tunnel for cases S1 and S2, respectively. The presence of the existing tunnel stiffens the soil and therefore reduces the ground surface settlement. The predicted $i x$ and $K$ values for ground surface settlement trough are larger for the case of the twin tunnel as compared to a single tunnel. 
(2) The effect of tunnelling on the existing tunnel for construction sequence S2 is more significant compared to case $\mathrm{S} 1$ in terms of both displacement in location and deformation in shape.

(3) The maximum bending moment occurs at the crown and invert for both cases S1 and S2, whereas the minimum bending moment occurs at the spring line for both cases. It is noted that the magnitudes of maximum and minimum bending moment for case S2 are larger than that for case $\mathrm{S} 1$ which explains the larger horizontal and vertical deformations of the existing tunnel for case S2 as compared to that for case S1.

(4) When the new tunnel face approaches the centre line of the existing tunnel, the transverse normal stresses at the crown for S1 and invert for S2 are transferred to the shoulder for case S1 and knee for case S2 in the transverse section. The maximum normal and shear stress change for case S2 is larger than that for case S1 which explains the larger deformation and bending moment induced on the existing tunnel for the case S2 as compared to that for S1.

(5) For both cases S1 and S2, the effect of stress release is significant on the existing tunnel when the advancing tunnel face is between a distance of 3D behind and 6D ahead of the existing tunnel center line.

The limitations of this study mainly include two aspects. First, the soil constitutive model adopted in the simulations did not account for soil small-strain $(0.001 \%$ to $1 \%)$ stiffness which is a key parameter for predicting ground deformations and dynamic responses of many earth structures such as retaining walls, foundations, and tunnels [21, 22]. Soil small-strain stiffness characterizes high stiffness of soil at initial small strains $(0.001 \%$ to $1 \%)$. If the soil constitutive model adopted in this study incorporates soil small-strain stiffness, the soils far away the tunnel experience a smaller deformation, while the soils near the tunnel experience a larger deformation. Second, the mechanical parameters for the dry sand and tunnel lining lack of a corresponding engineering background. In order to assist the engineering design, the further numerical study should be related with a specific practical project or model experiment about perpendicular crossing tunnelling, which also facilitates the validation and optimization of the numerical model for this problem.

\section{Data Availability}

The numerical data used to support the findings of this study are available from the corresponding author upon request.

\section{Conflicts of Interest}

The authors declare that they have no conflicts of interest.

\section{Acknowledgments}

This work was supported by the National Natural Science Foundation of China (51608477 and 51338009), Key
Research and Development Program of Zhejiang Province (2019C03111), Key Research and Development Program of Hebei Province (18276111D), Zhejiang Provincial Natural Science Foundation of China (LQ18E080004 and LQ17E080003), and Research Project of Power China Huadong Engineering Corporation Limited (kY2016-02-31).

\section{References}

[1] M. L. Cooper, D. N. Chapman, C. D. F. Rogers, and A. H. C. Chan, "Movements in the piccadilly line tunnels due to the heathrow express construction," Géotechnique, vol. 52, no. 4, pp. 243-257, 2002.

[2] R. J. Mair, R. N. Taylor, and J. B. Burland, "Prediction of ground movements and assessment of risk of building damage due to bored tunnelling," in Proceedings of the International Conference of Geotechnical Aspects of Underground Construction in Soft Ground, pp. 713-718, London, UK, July 1996.

[3] C. J. Lee, C. R. Wu, B. R. Wu, and S. H. Yang, "Impact on an existing tunnel due to nearby new tunneling," in Proceedings of the International Offshore and Polar Engineering Conferenc-e, vol. 12, pp. 660-665, Kitakyushu, Japan, May 2002.

[4] C. W. Ng, K. M. Lee, and D. K. Tang, "Three-dimensional numerical investigations of new Austrian tunnelling method (NATM) twin tunnel interactions," Canadian Geotechnical Journal, vol. 41, no. 3, pp. 523-539, 2004.

[5] A. M. Marshall and R. J. Mair, "Centrifuge modelling to investigate soil-structure interaction mechanisms resulting from tunnel construction beneath buried pipelines," in Proceedings of the International Symposium on Geotechnical Apects of Underground Construction in Soft Ground, pp. 703-707, Taylor And Francis Group, Shanghai, China, July 2009.

[6] T. E. Vorster, A. Klar, K. Soga, and R. J. Mair, "Estimating the effects of tunneling on existing pipelines," Journal of Geotechnical and Geoenvironmental Engineering, vol. 131, no. 11, pp. 1399-1410, 2005.

[7] Y. J. Lee and C. S. Yoo, "Behaviour of a bored tunnel adjacent to a line of loaded piles," Tunnelling and Underground Space Technology, vol. 21, no. 3-4, p. 370, 2006.

[8] S. H. Kim, H. J. Burd, and G. W. E. Milligan, "Model testing of closely spaced tunnels in clay," Géotechnique, vol. 48, no. 3, pp. 375-388, 1998.

[9] H. Y. Liu, J. C. Small, J. P. Carter, and D. J. Williams, "Effects of tunnelling on existing support systems of perpendicularly crossing tunnels," Computers and Geotechnics, vol. 36, no. 5, pp. 880-894, 2009.

[10] ABAQUS, Theory Manual, Version 6.3, Hibbitt, Karlson and Sorensen Inc, Pawtucket, RI, USA, 2004.

[11] P.-A. Von Wolffersdorff, "A hypoplastic relation for granular materials with a predefined limit state surface," Mechanics of Cohesive-Frictional Materials, vol. 1, no. 3, pp. 251-271, 1996.

[12] A. Niemunis and I. Herle, "Hypoplastic model for cohesionless soils with elastic strain range," Mechanics of Cohesive-Frictional Materials, vol. 2, no. 4, pp. 279-299, 1997.

[13] Y. Hong, M. A. Soomro, and C. W. W. Ng, "Settlement and load transfer mechanism of pile group due to side-by-side twin tunnelling," Computers and Geotechnics, vol. 64, pp. 105-119, 2015.

[14] K. W. D. Tang, "Numerical studies of multiple NATM tunnel interaction in soft ground," M.Phil. thesis, Department of Civil Engineering, Hong Kong University of Science and Technology, Hong Kong, China, 2001. 
[15] ACI, Building Code Requirements for Structural Concrete and Commentary (ACI 318M-11), American Concrete Institute (ACI), Farmington Hills, MI, USA, 2011.

[16] G. Wei, "Selection and distribution of ground loss ratio induced by shield tunnel construction," Chinese Journal of Geotechnical Engineering, vol. 32, no. 9, pp. 1354-1361, 2010, in Chinese.

[17] Q. Li and D. S. Zeng, "3D FEM deformation analysis with the new tunnel perpendicularly crossing under the old one," Rocks and Soil Mechanics, vol. 22, no. 3, pp. 334-338, 2001, in Chinese.

[18] C. W. W. Ng, T. Boonyarak, and D. Mašín, "Three-dimensional centrifuge and numerical modeling of the interaction between perpendicularly crossing tunnels," Canadian Geotechnical Journal, vol. 50, no. 9, pp. 935-946, 2013.

[19] T. Boonyarak and C. W. W. Ng, "Effects of construction sequence and cover depth on crossing-tunnel interaction," Canadian Geotechnical Journal, vol. 52, no. 7, pp. 851-867, 2014.

[20] K. Zhao, M. Bonini, D. Debernardi, M. Janutolo, G. Barla, and G. Chen, "Computational modelling of the mechanised excavation of deep tunnels in weak rock," Computers and Geotechnics, vol. 66, pp. 158-171, 2015.

[21] D. Mašín, "3D modelling of a NATM tunnel in high K0 clay using two different constitutive models," ASCE Journal of Geotechnical and Geoenvironmental Engineering, vol. 135, no. 9, pp. 1326-1335, 2009.

[22] K. S. Wong and D. Mašín, "Coupled hydro-mechanical model for partially saturated soils predicting small strain stiffness," Computers and Geotechnics, vol. 61, pp. 355-369, 2014. 


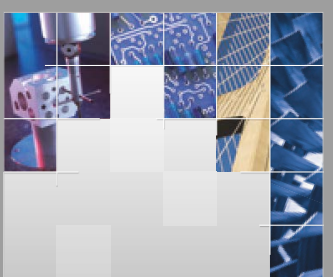

\section{Enfincering}
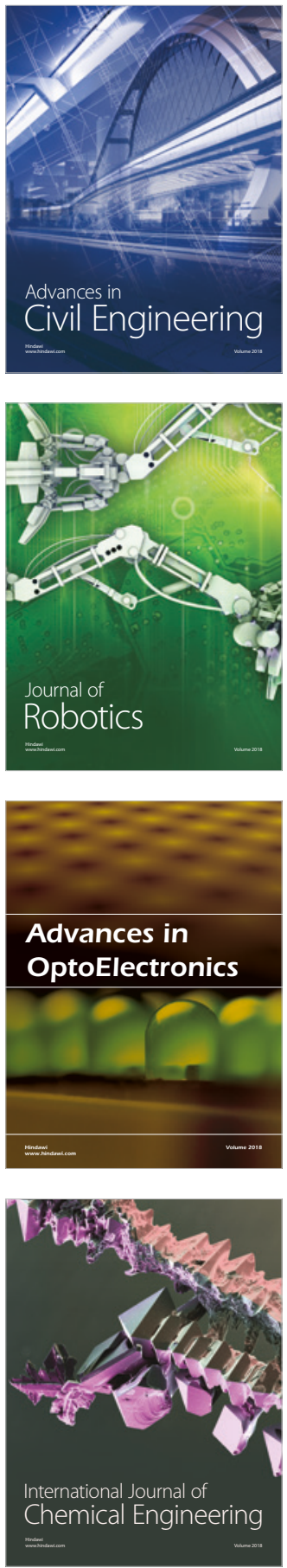

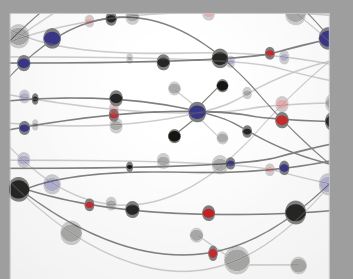

\section{Rotating \\ Machinery}

The Scientific World Journal

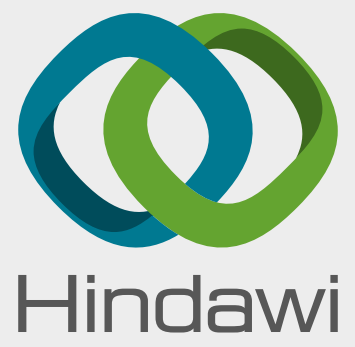

Submit your manuscripts at

www.hindawi.com
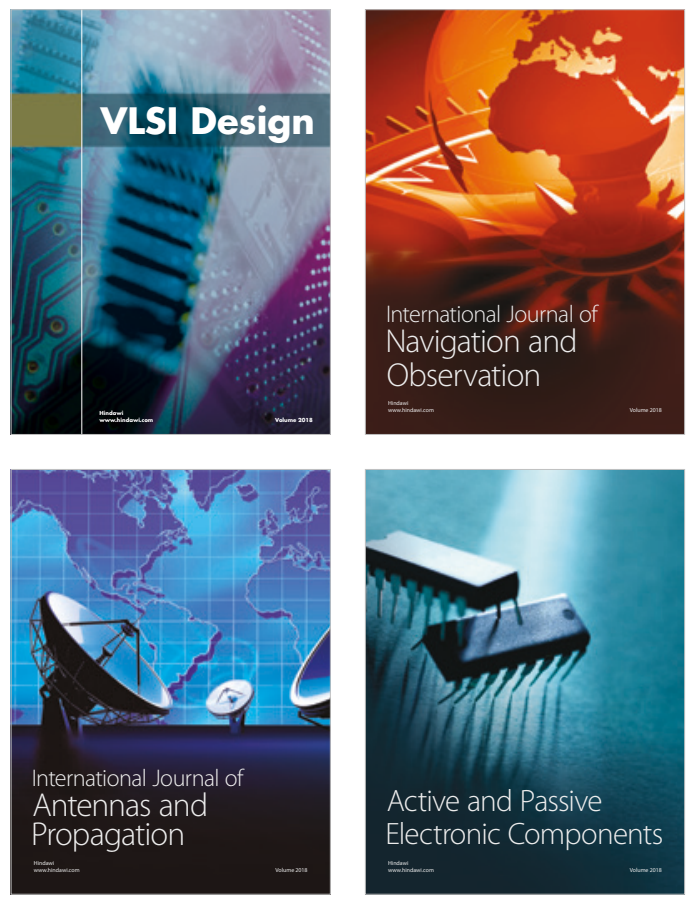
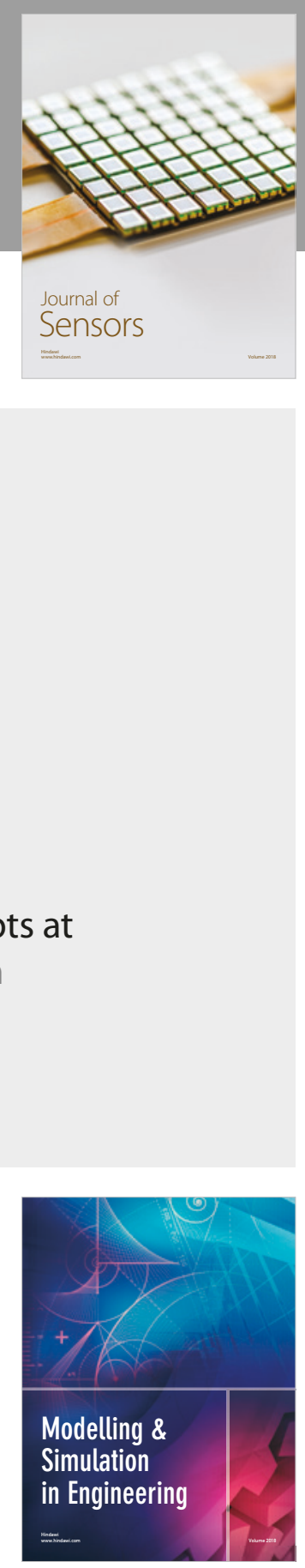

\section{Advances \\ Multimedia}
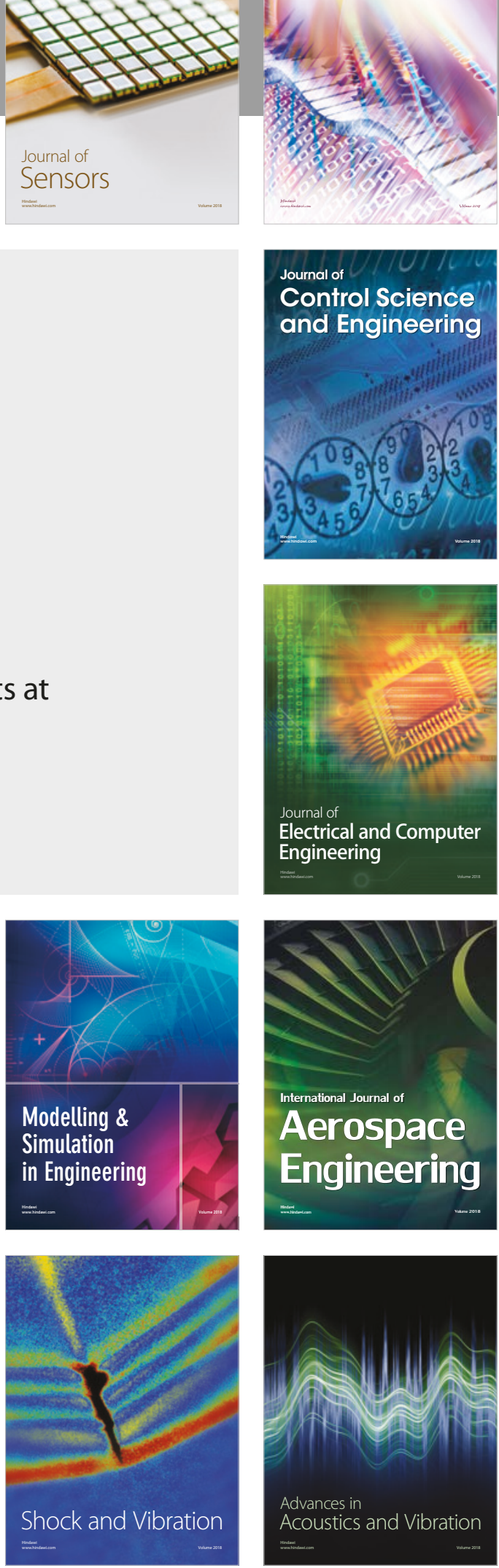